\title{
NEGOTIATION BASED ON PERSONALITY
}

\author{
Hong Zhang and Yuhui Qiu \\ Faculty of Computer and Information Science, Southwest China Normal University,Beibei, \\ Chongqing,,P.R.China,zhangh,yuqiu@swnu.edu.cn
}

\begin{abstract}
Negotiation is the highlight of e-commerce and artificial intelligence. This paper applies the idea of personality to BDI models and therefore attempts to present new negotiation architecture and to illustrate the protocol and algorithm. Through the experiments this paper analyses and proves that the personality (temperament) exerts great influence on concession rates in negotiation, and therefore affects the choices of negotiation strategy.
\end{abstract}

Key words: personality negotiation e-commerce BDI mode

\section{INTRODUCTION}

Software agent plays various roles in $\mathrm{BtoB}$ or $\mathrm{BtoC}$ commercial activity. Recently, people tend to use $\mathrm{CBB}$ model or BBT model to describe individual phases of e-commerce. In the two models mentioned, negotiation is an important link to which many researchers apply the advanced agent technology.

The negotiation in the e-commerce can be defined as the process of the transaction carried on by a group of agents to achieve a mutually acceptable agreement about the properties of some merchandizes. There also exists another definition in a stricter sense, that is, the process of deciding and reasoning supported by the artificial intelligence technology and mathematic technology (including logic, case based reasoning, belief correction optimization and game theory) and at the same time based on the agent's own inference of the belief, desire and intention. This demonstrates the importance of the psychological state to both sides of the negotiation during the process. Among the studies of the analysis and modeling of the agent's 
behavior, the BDI model (Belief, Desire and Intention) is one of the most representative ones. Belief and Desire represent the informational and evaluative state of the agent. While Intention represents the past decision of the agent, which is the key to the ideal performance under the limitation of given condition. A great deal of research work has been done on the form of the BDI model, focusing on describing its nature and behavior. The recognition of the agent's behavior is based on reasoning and goal driving (such as the intentional behavior). While the determining of the goal, the choosing and deciding of the parameter, the semantic and behavior are all based on his beliefs. Therefore the construction of the BDI model plays a very important role in the constructing negotiation models. However, it is regret that many models that had been recently constructed failed to relate the individual psychological factor to them. The present paper is attempting to explore the BDI model from this point.

\section{BDI MODEL BASED ON PERSONALITY}

The psychological process during the negotiation between the purchaser and the bargainer is the amalgamation and consolidation of the three processes of cognition, emotion, and consciousness, which are derived from the transformation of the properties of the goods into the currency. The psychological phenomenon of the behaviors between the purchaser and the bargainer is the psychological manifestation of an individual in a group as a "person", which is inevitably controlled by the individual psychological characteristics. In commercial activities a person can have various psychological activities - such as perception, apperception, memory, association, attention, imagination, thinking, will and so on, which exhibit the common rules of human psychological activities. As an individual, the person in commercial activities always acts as both purchaser and bargainer, no matter what might be the content in every specific negotiation. And he will keep those steady and essential psychological qualities in a unique combination. This is called consumer individuality. In the negotiation process this personality displays the disparity in the aspects of ability, character, temperament, interest and so on. Thus the difference in the behaviors of the negotiation process comes into being.

In commercial activities, the basis of the psychological process between the purchaser and bargainer includes the common process of psychological activities, consumers' individual psychology, consumption needs, and purchasing motives and so on. In the commercial activities of BtoB which are carried on the basis of a fixed relationship and in an atmosphere of friendliness, the need, certainly, is the precondition for the negotiation 
between the purchaser and bargainer; and therefore, their motivation are naturally favorable. Therefore, we hold that under such circumstance, personalities play a crucial role in the negotiation process.

The following is an observation of the situations of the BDI Model after the personalities are added to it. In the process of negotiation, the belief, desire and intention in it are always influenced by cognition, emotion and consciousness. And if we add the common personalities to the BDI Model (BDI-P), we can get the following graph (figure 1).

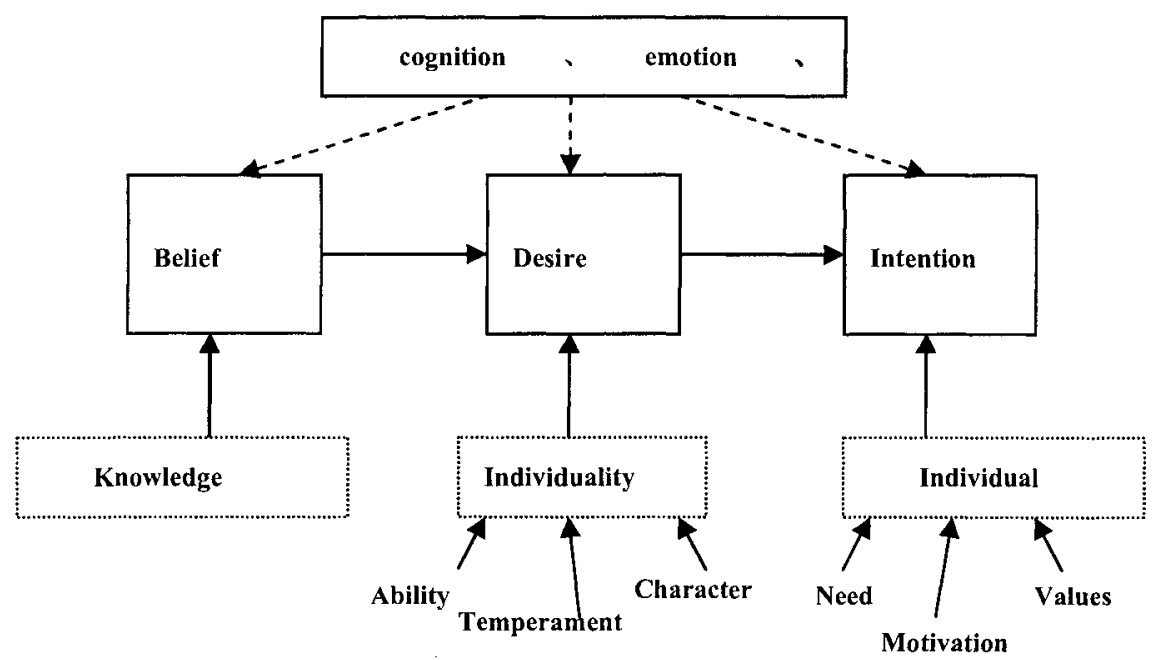

Figure 1. Figure 1 BDI mode based on Personality (BDI-P)

\section{CONCESSION RATE BASED ON TEMPERAMENT}

In this paper, two individual mental (temperament and character) factor will be added in experiments. Temperament as defined here refers to individual in behavioral style. Carl Jung asserted that people are fundamentally different and can be classified into "psychological types" based on their particular preferences. The four pairs of opposite preferences are Extraverting (E) and Introverting (I), Sensing (S) and intuiting $(\mathrm{N})$, Thinking (T) and Feeling (F), and Judging (J) and Perceiving (P). Within each pair of opposite preferences, a person leans toward one or the other in most cases

In various tactics (for seller agent), the predominant factor is personality of counter's agent, which decide which concession rate will be adopted. 


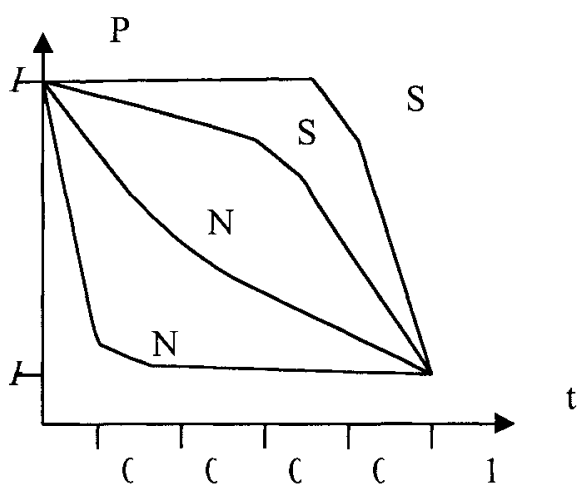

Figure 2. Various concession rate based on temperament

\section{TACTIC OF NEGOTIATION}

This tactic determines the recommended price based on the amount of time remaining and concession rate related time for the agent. . Assume that the agent is biding at time $0 \leq t \leq t_{\max }$. The agent bids from $P_{\max }$ as beginning price, when $t=0$, and at $t=t_{\max }$, the agent can't get reasonable price, negotiation is failed.

To calculate the bid value at time t, the following expressing is used:

$$
p(t)=F_{c r}(t) \times p_{\max }
$$

When $\mathrm{F}_{\mathrm{cr}}(\mathrm{t})$ is a polynomial function of he form:

$$
F_{c r}(t)=1-K_{c r}\left(t / t_{\max }\right) C R_{i}(t)
$$

$\mathrm{K}_{\mathrm{cr}}$ is a constant that determined the value of the end bid of the agent in negotiation. $C_{i}(t)$ is function relation to temperament. $i \in\{S J, S P, N T, N F\}$ By varying the value of $F_{c r}(t)$, a wide range of time dependant function can be defined from those that start biding near $\mathrm{p}_{\max }$, to those that only bid near $P_{\max }\left(1-\mathrm{K}_{\mathrm{cr}}\right)$, to all possibilities between. The only condition is that

$$
0 \leq F_{c r}(t) \leq 1, F_{c r}(0)=1, F_{c r}\left(t_{\max }\right)=1-K_{r t}, \text { and } 0 \leq k_{r t} \leq 1
$$

\section{CONCLUSION AND FUTURE WORK}

Psychologists have identified human temperament as predominant factor in the patterns of human behavior. Neuroscience research indicates that temperament is an innate property of the brain. The potential for employing human temperament as an effective negotiation is strong. The mode based 
on personality proposed a solution to characterize concession rate in negotiation by taking human factor, particularly human temperament into considerations. Our main line of work is to further explore the development of strategies for our negotiation agent. Since the process in which the negotiation in e-commerce is running is highly dynamic, we intend to extend this work by using a revolution method to classify the users, and develop various strategies for different temperament people.

\section{REFERENCES}

1. Keirsey, David, and Marilyn Bates, "Please Understand Me,"Del Mar, Ca: Prometheus Nemesis Books, 1978.

2. P. Faratin, C. Sierra and N. R. Jennings (2000) "Using similarity criteria to make negotiation trade-offs" Proc. 4th Int. Conf on Multi-Agent Systems, Boston, 119-126.

3. P. Faratin, C. Sierra, and N. R. Jennings (1998) "Negotiation decision functions for autonomous agents" Int. J. of Robotics and Autonomous Systems 24 (3-4) 159-182.

4. N. R. Jennings, P. Faratin, A. R. Lomuscio, S. Parsons, C. Sierra and M. Wooldridge(2001) "Automated negotiation: prospects, methods and challenges" Int. J. of GroupDecision and Negotiation 10 (2) 195-215. 